\title{
Neural correlates of fluid reasoning in children and adults
}

\author{
Samantha B. Wright ${ }^{1, \dagger}$, Bryan J. Matlen ${ }^{2,}$, Carol L. Baym ${ }^{3}$, Emilio Ferrer ${ }^{4}$ and Silvia A. Bunge ${ }^{1,5, *}$ \\ 1. Helen Wills Neuroscience Institute, University of California at Berkeley, Berkeley, CA, USA \\ 2. Department of Psychology, Carnegie Mellon University, Pittsburgh, CA, USA \\ 3. Department of Psychology, University of Illinois at Urbana-Champaign, Champaign, IL, USA \\ 4. Department of Psychology, University of California at Davis, Davis, CA, USA \\ 5. Department of Psychology, University of California at Berkeley, Berkeley, CA, USA
}

Edited by: Robert T. Knight, University of California Berkeley, USA

Reviewed by: Anna C. Nobre, University of Oxford, UK

Donald T. Stuss, Baycrest Centre for Geriatric Care, Canada; University of Toronto, Canada

\begin{abstract}
Fluid reasoning, or the capacity to think logically and solve novel problems, is central to the development of human cognition, but little is known about the underlying neural changes. During the acquisition of event-related fMRI data, children aged $6-13(N=16)$ and young adults $(N=17)$ performed a task in which they were asked to identify semantic relationships between drawings of common objects. On semantic problems, participants indicated which of five objects was most closely semantically related to a cued object. On analogy problems, participants solved a visual propositional analogy (e.g., shoe is to foot as glove is to...?) by indicating which of four objects would complete the problem; these problems required integration of two semantic relations, or relational integration. Our prior research on analogical reasoning in adults implicated left anterior ventrolateral prefrontal cortex (VLPFC) in the controlled retrieval of individual semantic relationships, and rostrolateral prefrontal cortex (RLPFC) in relational integration. In this study, age-related changes in the recruitment of VLPFC, temporal cortex, and other cortical regions were observed during the retrieval of individual semantic relations. In contrast, agerelated changes in RLPFC function were observed during relational integration. Children aged 6-13 engage RLPFC too late in the analogy trials to influence their behavioral responses, suggesting that important changes in RLPFC function take place during adolescence.
\end{abstract}

Keywords: development, cognition, brain, analogy, relational, functional magnetic resonance imaging, hierarchy, frontal

\section{INTRODUCTION}

Fluid reasoning represents the capacity to think logically and solve problems in novel situations. This construct is central to theories of human intelligence (Carroll, 1997; Cattell, 1987; Gray et al., 2003; Horn, 1988; Horn and Cattell, 1967; McArdle and Woodcock, 1998). In Iongitudinal studies, fluid reasoning ability has been identified as a leading indicator of changes in crystallized abilities (McArdle, 2001), as well as changes in academic achievement factors such as quantitative ability, academic knowledge (Ferrer and McArdle, 2004), and reading (Ferrer et al., 2007). The strongest influences of fluid reasoning ability on later achievement have been observed among children of ages 5 to 10 (Ferrer and McArdle, 2004; Ferrer et al., 2007), although substantial but weaker effects are apparent until age 24. These findings suggest that the development of fluid reasoning serves as a scaffold that allows a child to acquire other abilities (Blair, 2006; Cattell, 1971, 1987). As such, the development of reasoning ability is central to cognitive development from childhood to early adulthood.

\footnotetext{
*Correspondence: Silvia A. Bunge, Helen Wills Neuroscience Institute \& Department of Psychology, University of California at Berkeley, 132 Barker Hall, MC \#3190 Berkeley, CA 94720-3190, USA. e-mail: sbunge@berkeley.edu

Shared first-authorship.

Received: 19 September 2007; paper pending published: 29 November 2007; accepted: 30 January 2008; published online: 28 March 2008.

Citation: Front. Hum. Neurosci. (2008) 1: 8. doi: 10.3389/neuro.09.008.2007

Copyright (c) 2008 Wright, Matlen, Baym, Ferrer and Bunge. This is an open-access article subject to an exclusive license agreement between the authors and the Frontiers Research Foundation, which permits unrestricted use, distribution, and reproduction in any medium, provided the original authors and source are credited.
}

One form of fluid reasoning is relational reasoning: that is, the ability to find correspondences between the structures of distinct mental representations. Analogical reasoning may be considered a specialized form of relational reasoning, one that entails abstracting a higher-order relation between a familiar representation (the base) and mapping it to a novel representation (the target) (Gentner, 1988; Goswami and Brown, 1989). The most rudimentary form of analogical reasoning is captured by propositional analogy problems (e.g., car is to road as boat is to...?) In these problems, it is necessary to not only abstract the higher-order relationship between the base items (car "moves on" road) but also map this higher-order relationship to the target pair (boat "moves on" ?) in order to fill in the missing piece (water).

Piaget argued that, before the stage of formal operations, around age 11 , children do not possess the cognitive capacity to represent the necessary relations needed to perform classical analogy problems (Inhelder and Piaget, 1958). When Piaget and his colleagues presented children with pictorial problems of the form 'A:B::C:?' and asked them to find the $D$ term in a pictorial set, he found that children often relied on lower-order relations to solve the problems, choosing items that were associated with or looked like the C item (Piaget et al., 1977). Sternberg and colleagues found similar limitations in young children's analogical reasoning, observing an over-reliance on lower-order relations during analogical problem solving (Sternberg and Downing, 1982; Sternberg and Nigro, 1980).

More recent investigations have revealed that children can effectively reason by analogy at young ages. Children as young as 3 years of age have been found to perform successfully on classical ' $A: B:: C: D$ ' analogies as long as they are familiar with the relevant relations necessary to solve the problems (Goswami and Brown, 1989). Numerous other studies have demonstrated children's ability to solve problems through analogical 
reasoning (Holyoak et al., 1984; Pierce and Gholson, 1994; Richland et al., 2006; Singer-Freeman and Goswami, 2001; Tunteler and Resing, 2002), and prominent analogical theorists have suggested that analogy is an essential means by which cognition develops (Brown, 1990; Goswami and Brown, 1989).

Little is known about the neural underpinnings of development in analogical reasoning, or more generally fluid reasoning, although it is widely assumed that such development is linked to the maturation of prefrontal cortex (PFC). Based on prior studies in adults (Braver and Bongiolatti, 2002; Bunge et al., 2005; Christoff et al., 2001, 2003; Green et al., 2006; Wendelken et al., 2007), we hypothesized that agerelated improvements in reasoning would rely in part on changes in the recruitment of anterior prefrontal cortex, or more specifically rostrolateral PFC (RLPFC; lateral Brodmann area 10, extending to the most anterior parts of $B A 11,9$, and/or 46), during the joint consideration of multiple relations. Our group has recently provided evidence for age-related changes in the involvement of PFC - in particular, RLPFC - on the Raven's Progressive Matrices (RPM), a standard fluid reasoning task that requires minimal domain-specific knowledge (Crone et al., in press).

In the present study, we sought to examine the neural correlates of age-related differences in analogical reasoning on a propositional analogy task. We had previously conducted two studies in which adults were asked to evaluate verbal propositional analogies, such as 'car is to road as sailboat is to water' (Bunge et al., 2005; Wendelken et al., 2007). On the basis of these findings we argued that, while left anterior ventrolateral PFC (VLPFC; BA 45/47) was involved in the controlled retrieval of relationships between individual items (e.g., the way in which a car is associated with a road), left RLPFC (BA 10/11) was involved in jointly considering two relations to evaluate a propositional analogy. We proposed a hierarchical relationship between these regions, such that RLPFC integrates the products of VLPFC (see also Christoff et al., 2003; Bunge and Zelazo, 2006).

Given this hypothesized hierarchy in the functional organization of PFC, we predicted that RLPFC should exhibit adult-like activation on a propositional analogy task later in development than VLPFC. To test these predictions, we collected fMRI data for children and young adults during performance of a propositional analogy task involving drawings of common objects. The children's group included participants ranging in age from 6 to 13, enabling us to consider age as a continuous variable for this period of important cognitive changes.

\section{MATERIALS AND METHODS Participants}

Data from 16 typically developing $6-13$-year olds $(7 \mathrm{M}, M=9.87$, $\mathrm{SD}=2.09$; mode $=10)$ and 17 adults aged 19-26 (6 M, $M=22.05$ $\mathrm{SD}=2.09$; mode $=20$ ) were included in the analyses. Each age between 6-13 and 19-26 was represented in the sample. An additional seven children participated in the study but were excluded from analysis due to excessive head movement during scanning. All children and adults were recruited from the Davis and Sacramento areas. Participants were compensated \$10 per hour for their participation. Informed consent was acquired from all participants in accordance with the Institutional Review Board at the University of California at Davis.

\section{Procedure}

Stimuli. Our visual propositional analogy task (Figure 1) was based on a test from the Kaufman Brief Intelligence Test, $2^{\text {nd }}$ edition (KBIT-2) designed for use in children. We designed over 80 pictorial analogies with Adobe Photoshop, making use of line drawings from "The Big Box of Art: 1 Million". All stimuli were pictures of common objects known to young children, as judged by age-of-acquisition psycholinguistic norms for the words that they depicted (Gilhooly and Logie, 1980).

Task conditions. The task included two conditions: semantic and analogy problems. On semantic problems (Figure 1A), participants saw one

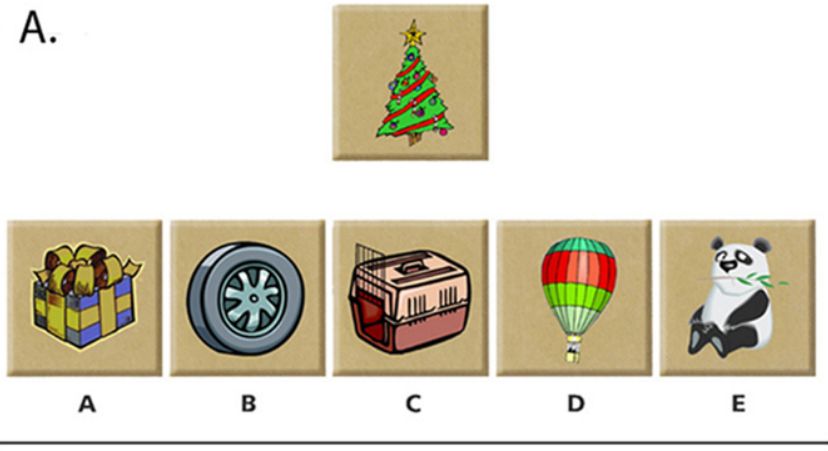

B.

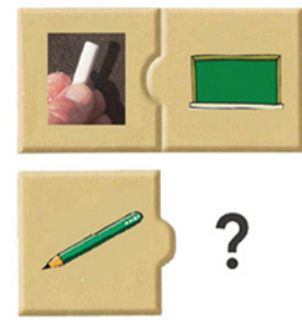

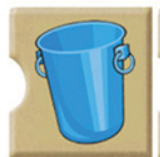

A

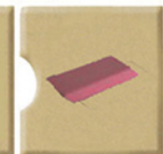

B

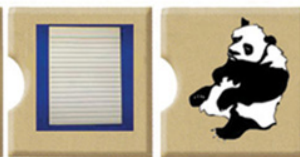

C

D
Figure 1. A sample problem is displayed for each of the two task conditions. (A) On semantic problems, one must choose from four images the object that is most closely related to the target image. (Correct answer: A; $D=$ perceptual lure). (B) On analogy problems, one must consider the relationship between the two images above, and identify the image below that completes the analogy (Correct answer: $C$; $B=$ semantic lure).

target stimulus (e.g., a Christmas tree), and were asked to indicate with a button press which of five choice stimuli went best with it (e.g., a wrapped present). In our version of the task, each semantic trial included a perceptual lure - i.e., a drawing that was perceptually similar to the target stimulus in terms of color or shape, but was not otherwise closely related (in this example, a hot-air balloon in the same colors as the Christmas tree). In this way, we could gauge the extent to which children tended to respond on the basis of their knowledge about an item rather than simply on the basis of surface similarity.

On analogy problems (Figure 1B), participants saw three target stimuli, and were asked to indicate with a button press which of 4 figures best completed the array (e.g., chalk is related to a chalkboard as a pencil is related to...? paper). In our version of the task, half of the analogy trials included a perceptual lure that was perceptually similar to the third target stimulus. The other half of the analogy trials include a semantic lure -i.e., a drawing of an object (e.g., an eraser) whose meaning was related to the third target stimulus (a pencil), but not in a way that was analogous to the relationship between the first and second target stimuli (chalk and a chalkboard). In this way, we could examine whether children correctly identified analogous relationships, or whether they were lured by perceptual or semantic relatedness.

\section{Training procedure}

Children were introduced to the scanner environment with a mock scanner, where they were trained to lie still. Prior to fMRI data acquisition, all participants were provided with explicit task instructions, and were 
asked to answer six analogy problems on paper (three semantic and three analogy problems). Participants were given explicit instructions to help them avoid committing lure answer choices, such as, "just because some of the answers look the same, it doesn't always mean they are the right answer." Once in the actual scanner, participants practiced using the button box to respond to the sample problems.

\section{Data acquisition}

fMRI task procedure. Participants performed a total of 40 semantic and 40 analogy trials during fMRI data acquisition. The task was split up into 4 runs, each containing 20 trials (ten from each condition), with each run lasting 4.5 minutes. Run lists were presented in a randomized order, and participants were given up to 12 seconds to respond on each trial. The picture remained on the screen until the subject pressed a button or until the duration of the trial had ended.

MRI data acquisition. Imaging was performed using an 8-channel phased-array coil on a 3-Tesla Siemens Trio MRI scanner (Siemens Medical Solutions, Erlangen, Germany) at the University of California at Davis Imaging Research Center (Sacramento, CA). Participants viewed visual stimuli on a projection screen using an angled mirror and responded using a button box in the right hand.

After acquisition of a T2 localizer scan, four functional runs were collected $(\mathrm{TR}=2000 \mathrm{~ms}, \mathrm{TE}=25 \mathrm{~ms}, 34$ axial slices, no interslicegap, $3.4 \times 3.4 \times 4 \mathrm{~mm}$ voxels, flip angle $=90^{\circ}$, field of view $=220 \mathrm{~mm}$, 135 volumes per run). A gradient-echo echo-planar pulse Prospective Acquisition Correction (3D-PACE) sequence was used to minimize motion artifacts by prospectively adjusting scan parameters throughout a run on the basis of real-time assessment of head motion (Siemens Medical Solutions; Thesen et al., 2000). Four volumes from the start of each functional scan were removed from analysis to account for magnetic field equilibration. Following the functional scans, high-resolution threedimensional T1 MPRAGE anatomical images were acquired.

\section{fMRI data preprocessing and analysis}

Preprocessing. fMRI data were analyzed using SPM2 (Wellcome Department of Cognitive Neurology, London, UK). Functional volumes from each participant were corrected for interleaved slice acquisition, and then were translated using a rigid-body motion correction. Functional images were then normalized to an EPI template using a 12-parameter affine transformation and resampled to $3 \times 3 \times 4 \mathrm{~mm}$ voxels. The SPM EPI template has been validated for use in normalization of brain volumes for children aged 6 and up (Burgund et al., 2002; Kang et al., 2003). After normalization, functional images were smoothed using an $8 \mathrm{~mm}$ fullwidth at half maximum isotropic Gaussian kernel.

Individual subject analyses. Statistical analyses were performed using the general linear model in SPM2. fMRI time-series data were modeled as a series of semantic and analogy events, time-locked to the onset of each trial, and were convolved with a canonical hemodynamic response function. The resulting functions were used as covariates in a general linear model, along with a basis set of cosine functions that high-pass filtered the data, as well as a covariate for session effects. Six motion regressors (indexing translation and rotation in the $x, y$, and $z$ dimensions for each two-second TR) were included in the analysis as covariates of no interest. The least-squares parameter estimates of height of the best-fitting synthetic HRF for each condition were used in pair-wise contrasts, and the resulting contrast images, computed on a subject-by-subject basis, were submitted to group analyses. Incorrect trials were modeled separately and were not included in analyses. Participants who moved more than $5 \mathrm{~mm}$ within a scan (i.e., seven children) were excluded from the study.

Group statistics. Three analytic approaches were used to characterize brain activation in children and adults on the visual analogy task: (1) whole-brain $t$-tests, (2) whole-brain multiple regression analyses including age and accuracy as regressors, and (3) region-of-interest (ROI) analyses, performed to fully characterize the activation profile of regions of a priori interest identified from the whole-brain maps.

In our prior event-related fMRI studies, we have found that children exhibit greater inter-individual variability in brain activation than adults, and therefore the whole-brain statistical thresholds commonly used in adult event-related fMRI studies ( $p<0.001$ uncorrected, or even $p<0.05$ corrected) were deemed overly stringent for exploratory analyses. As such, the whole-brain contrasts and multiple regressions were thresholded at $p<0.001$ uncorrected for adults and $p<0.005$ for children, with an extent threshold of 10 contiguous voxels.

\section{RESULTS}

\section{Behavioral results}

To assess participants' performance on our visual analogy task, two separate 2 (Age: children, adults) $\times 2$ (Condition: semantic, analogy) mixed analyses of variance (ANOVAs) were conducted, one for accuracy and one for response times (RTs) for correctly performed trials. Both age groups performed significantly above chance on all conditions, where chance performance was equivalent to $20 \%$ accuracy on semantic problems and 25\% accuracy on analogy problems (Figure 2).

The ANOVA focusing on proportion of correct responses revealed significant main effects of Age, $F(1,31)=12.8, p<0.005$, and Condition, $F(1,31)=76.4, p<.001$, as well as a significant two-way interaction, $F(1,31)=9.3, p<.01$, such that children made disproportionately more errors on analogy problems than did adults (Figure 2). The ANOVA focusing on RTs also revealed main effects of Age, $F(1,31)=52.1, p<0.001$, and Condition, $F(1,31)=451.0, p<0.001$, as well as a significant interaction between these two factors, $F(1,31)=5.8, p<0.05$, albeit less pronounced than for accuracy. These findings indicate that children exhibited a greater decrement in performance on analogy problems relative to semantic problems than adults.

To test for differences in performance on analogy trials involving perceptual vs. semantic lures, we conducted 2 (Age: children, adults) $\times 2$ (Lure Type: perceptual, semantic) mixed ANOVAs. These trial types did not differ in terms of accuracy (main effect of Lure Type: $F<1$; Lure Type $\times$ Age interaction: $F<1$ ). In terms of RTs, there was a significant main effect of lure type, $F(1,31)=14.2 ; p=0.001$, as well as a significant lure $\mathrm{x}$ group interaction, $F(1,31)=5.0 ; p=0.033$. Children responded more slowly on analogy trials that included a semantic lure than those that included a perceptual lure $(6.2 \pm 1.0$ seconds vs. $5.8 \pm 0.9)$; a marginal effect of lure type was also observed for adults ( $4.1 \pm 0.9$ vs. $4.0 \pm 0.9$ seconds). Thus, children, and to some extent adults, were particularly distracted by items that did not complete the analogy but were semantically related to one of the items in the stimulus array.
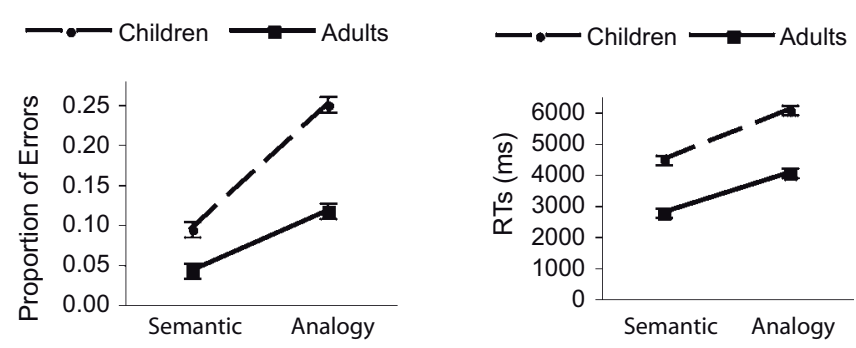

Figure 2. Behavioral results for performance during fMRI data acquisition are shown here. Proportion of errors and RTs for correctly performed trials are plotted separately for semantic and analogy conditions, and for adult and child groups. Error bars represent the within-subject error of the Condition $\times$ Group interactions. Both age groups showed an increase in errors and RTs for analogy compared to semantic trials. However, children made disproportionately more errors on analogy trials than adults. 
Previous studies have provided evidence of sex differences in fluid reasoning ability. Some work has shown that girls outperform boys in childhood and that this sex difference reverses in adolescence (e.g., Lynn et al., 2004). A univariate analysis of variance measuring the effect of sex and age on accuracy resulted in a significant effect of age $(F(1,32)=18.4, p<0.001)$ and a significant interaction between the two factors $(F(1,32)=6.6, p=0.016)$. To further characterize the effect of age, one-way ANOVAs were run separately for each group comparing accuracy and sex. While the adult group exhibited no significant performance difference between sexes $(F(1,16)=2.5, p>0.10)$, the child group did show a trend in which females displayed slightly better performance overall $F(1,15)=4.27, p=0.058)$. There was no systematic difference in age between the males and females $(F(1,14)=0.211, p=0.653)$. Given the small sex effect in this relatively small sample size, we did not examine these effects further with fMRI.

\section{Whole-brain fMRI analyses}

Whole-brain contrasts. To assess qualitatively whether children and adults engaged similar brain regions during task performance, exploratory whole-brain contrasts were generated for semantic trials relative to the fixation baseline, and for analogy > semantic trials (see Figure S1). On the whole, children appeared to exhibit a subset of the regions engaged by adults, rather than a distinct set of regions. In adults, the contrast of semantic $>$ fixation revealed activation in bilateral dorsolateral PFC (DLPFC; BA9), right VLPFC (BA 45), and right inferior parietal lobule (IPL; BA40), as well as premotor and visual cortices and basal ganglia. The analogy $>$ semantic contrast revealed bilateral VLPFC (BA 47), as well as additional activation in parietal and visual cortices and basal ganglia. In children, considerably fewer areas of activation were revealed, even at the more liberal threshold of $p<0.005$ uncorrected, likely reflecting greater inter-individual variability. The semantic $>$ fixation contrast revealed activation in DLPFC and premotor cortex (BA 9/6), and parietal (BA 7) and visual cortex. The analogy > semantic contrast revealed additional activation in bilateral premotor cortex and right visual/visual association cortex. Visual cortex is thought to be additionally activated in children since this group as a whole responded more slowly, and as a result saw the images for a longer period of time.

Whole-brain multiple regression analyses. No RLPFC activation was observed for the group contrast of analogy > semantic for either children or adults. Nonetheless, we sought to test whether some individuals recruited RLPFC during relational integration, and whether RLPFC activation correlated with age and/or performance. To this end, we computed whole-brain multiple regression analyses separately for the two groups, including age and accuracy as regressors for analogy trials. We sought to determine whether levels of activation on analogy trials relative to a resting baseline, and/or differential activation on analogy vs. semantic trials would be correlated with age and/or accuracy.

Our first multiple regression analysis revealed a region in left RLPFC that was increasingly engaged on analogy trials relative to fixation as a function of age among the children (Figure $3 \mathrm{~A}$ ). No significant correlations were observed for adults in this analysis.

Our second regression analysis identified regions for which there was a positive correlation between activation for analogy $>$ semantic with age and/or accuracy on analogy trials. No sizeable correlations were observed for children, but adults exhibited a positive correlation with accuracy for a region in right RLPFC. This region was located dorsally to the regions in left RLPFC identified from the other multiple regression analysis ( $Z$-coordinate $=+15$ vs. $0-4$ ).

Additional multiple regression analyses were aimed at identifying regions separately in children and adults for which semantic $>$ fixation activation was positively correlated with age or accuracy. Children exhibited positive correlations with age in a number of regions, including bilateral RLPFC and bilateral VLPFC.

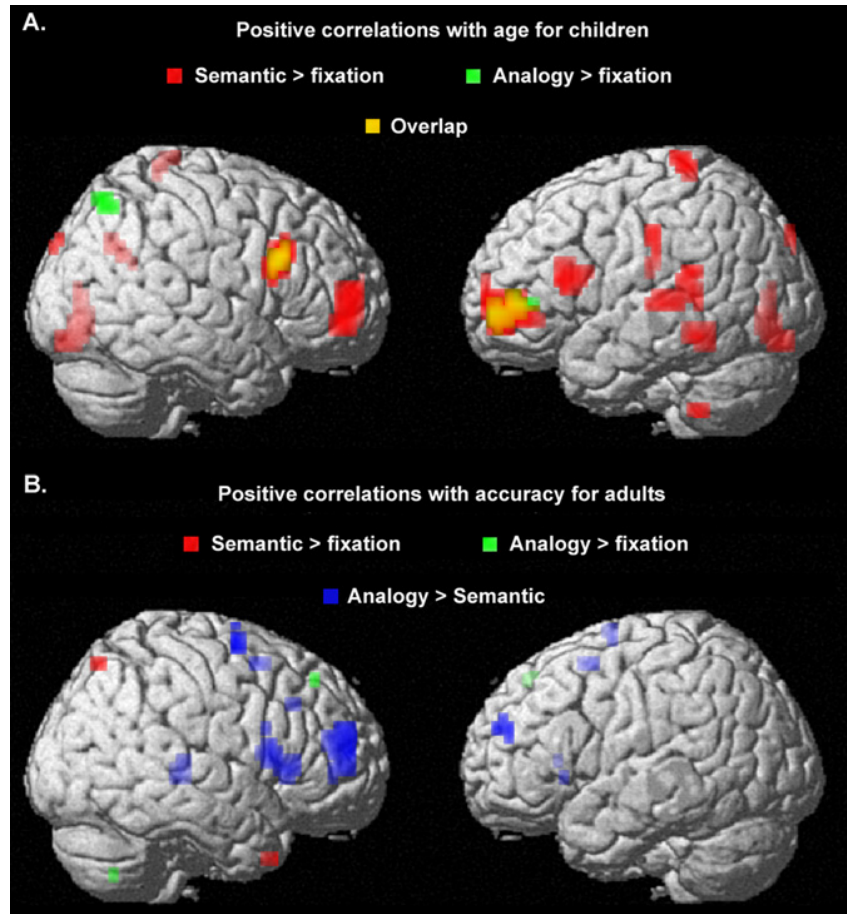

Figure 3. Whole-brain multiple regression analyses are displayed here. (A) Positive correlations with age from 6-13 were observed for semantic trials (shown in red) in a number of regions, including left RLPFC (BA 10; -27, $50,0)$, left VLPFC (BA 45;-42, 18, 16) and right DLPFC (BA 9; -48, 18, 26), and regions in temporal, parietal, motor, and visual regions. For analogy trials, positive correlations with age from 6-13 were observed only in left RLPFC, right VLPFC, and right superior parietal lobule (BA 7; 36, -66, 52). (B) Adults who exhibited higher accuracy on analogy trials exhibited greater differential activation between analogy and semantic trials in bilateral VLPFC and RLPFC, as well as several other regions. The most prominent cluster was observed in right $R L P F C(B A 10 ; 27,45,16)$.

\section{ROI analyses: VLPFC and RLPFC}

ROI identification and analyses. ROI analyses were conducted to further examine the activation profiles of the regions in left VLPFC and bilateral RLPFC identified from the multiple regression analyses displayed in Figure 3. An additional ROl analysis was performed on a functionally derived region in left motor cortex, to determine at which point during the trials activation in the PFC regions peaked relative to the motor response. Median timecourses for correctly performed semantic and analogy trials are displayed in Figures 4 and 5. Because RLPFC activation was substantially delayed relative to the onset of a trial, we found that the timecourses did not return to baseline until time 2 of the next trial. Thus, in displaying and analyzing the PFC timecourses, we omitted the first timepoint and set the second timepoint to a value of zero. The BOLD activation timecourse associated with each condition was extracted for each ROI and age group. A 2 (Condition: semantic, analogy) $\times 9$ (Timepoints: $2-18$ seconds) $\times 2$ (Group: children, adults) mixed ANOVA was computed for each ROI. Main effects and interactions involving age group and/or condition are discussed below.

Left VLPFC. Children and adults both exhibited task-related activation in left VLPFC relative to baseline. While this region failed to show a significant effect of or interaction involving Condition, there was a significant Timepoint $\times$ Group interaction, $F_{(8,248)}=2.3, p=0.022$, that prompted us to look separately at the two age groups for the Condition $\times$ Timepoint interaction that appeared to be present in the graph of the adult timecourse. 

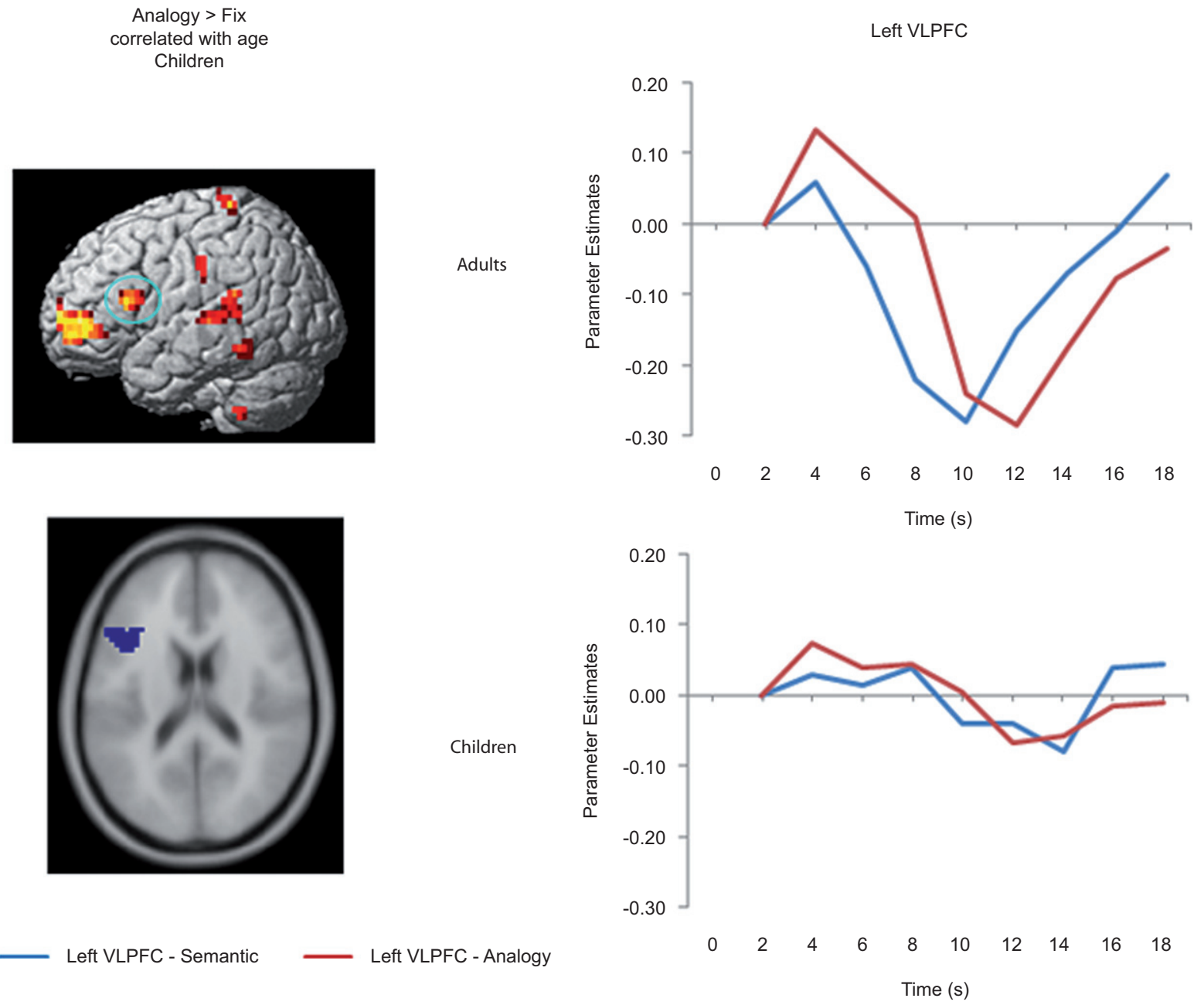

Figure 4. Left VLPFC ROI and timecourse. The left side displays the left VLPFC ROI identified from the positive correlation between semantic $>$ fixation activation and age in children (ROI circled in blue on the rendered image, and shown below on an axial slice). The right side displays the median timecourse of activation for this region. Semantic (blue) and analogy (red) trials are shown separately, for both adult and child groups.

As predicted, the children failed to show this interaction, $F<1$, while the adults showed a strong interaction, $F_{(8,128)}=3.5, p=0.001$, indicative of the effect of analogy $>$ semantic trials present at specific points in the left VLPFC timecourse for adults.

Bilateral RLPFC. Bilateral RLPFC exhibited marked differences in the timecourse of activation between age groups (Figure 5). Children exhibited delayed activation in RLPFC, peaking at 10 seconds - a full 4 seconds after the adults, despite the fact that RT differences between the groups were not this large.

We sought to determine whether the peak RLPFC activation in children occurred before or after the motor response. Because participants selected responses with their right hand, we compared the RLPFC timecourses to that of left motor cortex. For adults, the RLPFC activation peaked at around the same time as motor cortex, consistent with the idea that RLPFC contributes to performance. For children, in contrast, RLPFC peaked after motor cortex, suggesting that this region was not engaged in time to influence the behavioral response. Arguing against the possibility that children merely have a sluggish hemodynamic response in RLPFC, we have observed similar timecourses in this region for 8 to 12-year-olds and young adults in the context of a RPM task (Crone et al., in press).
Analysis of the RLPFC timecourses confirmed the presence of a Group $\times$ Timepoint interaction for both left and right RLPFC (left: $F_{(8,248)}=3.71, p<0.001$; right: $\left.F_{(8,248)}=4.521, p<0.001\right)$. Right RLPFC additionally exhibited a Condition $\times$ Timepoint interaction, $F_{(8,248)}=3.585$, $p=0.001$, qualified by a Group $\times$ Condition $\times$ Timepoint interaction, $F_{(8,248)}=2.653, p=0.008$. Post-hoc analyses indicated that condition type did not affect peak latency in either age group: for both analogy and semantic trials, RLPFC activation peaked at 6 seconds for adults and at 10 seconds for children.

\section{DISCUSSION}

Our behavioral results indicate that children aged 6-13 had little difficulty identifying the item most semantically related to the cue stimulus on semantic trials, even in the face of distraction from stimuli that were perceptually similar to the cue. Children in this age range were also, on the whole, capable of identifying analogous relationships between pairs of stimuli on analogy problems, even in the face of distraction from stimuli that were either perceptually or semantically related to one of the items in the cue stimulus array. Children did, however, make disproportionately more errors on analogy than semantic trials relative to adults. They were also disproportionately slower than adults on analogy trials that included semantic as opposed to perceptual lures. These results are consistent 

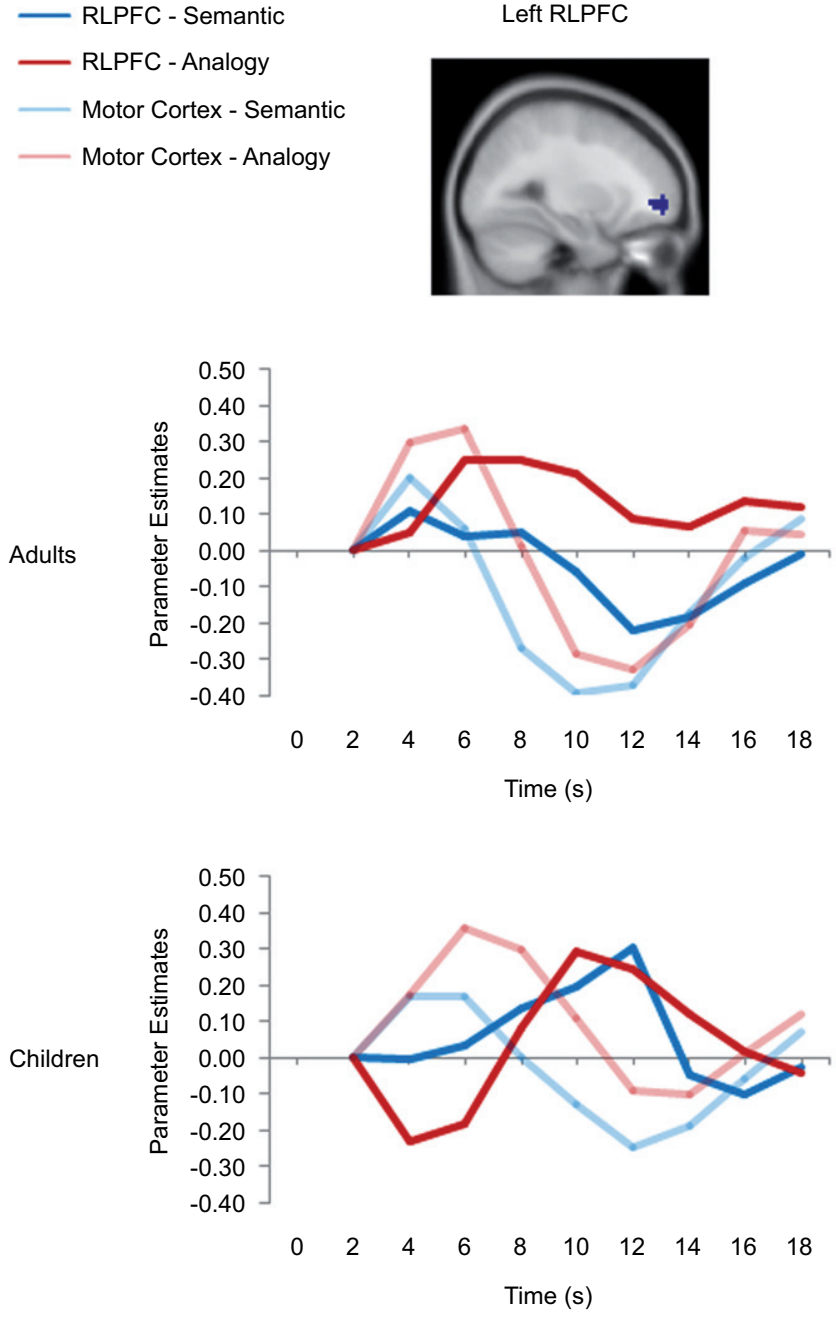

Right RLPFC
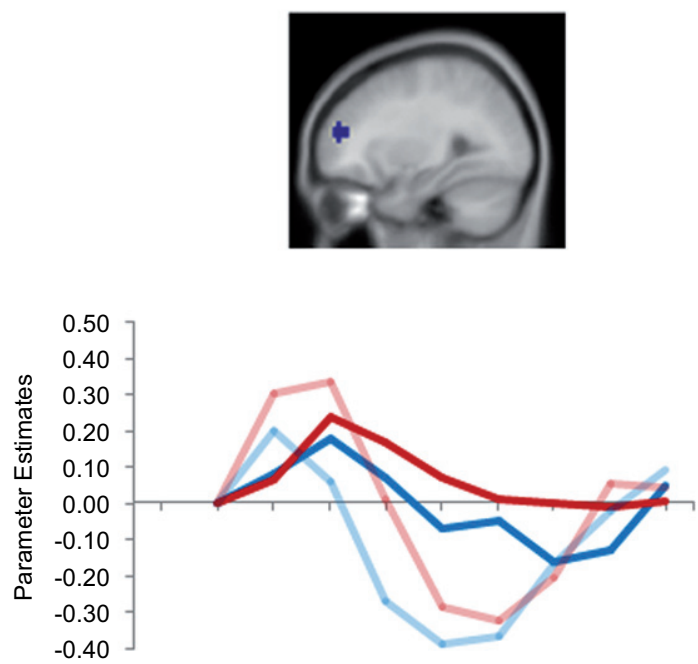

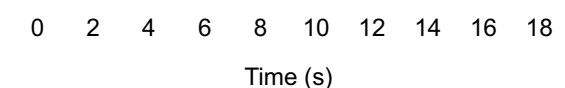

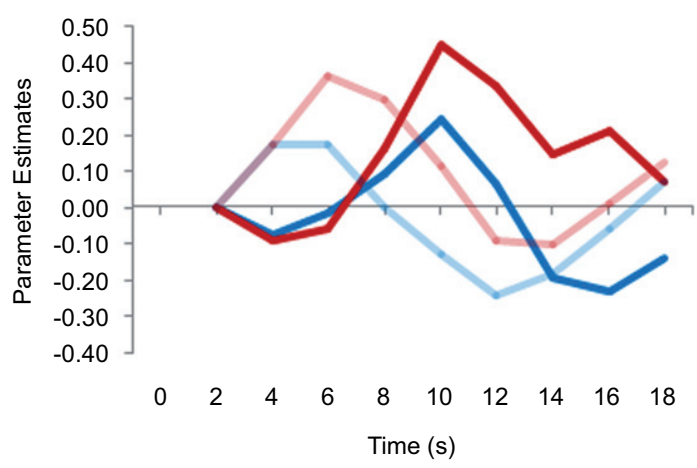

Figure 5. RLPFC ROIs and timecourses. The left side of the image displays a sagittal view of the left RLPFC ROI, and two separate timecourses displaying semantic and analogy activation every two seconds from where it is baselined at 2 seconds until 18 seconds after trial onset. The right side displays the right RLPFC region and timecourses. All timecourses also model the motor cortex ROI. Note that while in adults the RLPFC timecourses peak during the same 4-6-second period as the motor cortex ROI, in children these ROls peak much later at 10 to 12 seconds after trial onset.

with prior behavioral research in showing that children have greater difficulty integrating two relations on relational reasoning tasks, including analogical reasoning tasks (Crone et al., in press; Gentner, 1988; Holyoak et al., 1984; Richland et al., 2006).

We sought to test for differences between children and adults in left VLPFC, a region associated with controlled semantic retrieval (Bunge et al., 2005; Wagner et al., 2001), and in RLPFC, a region associated with relational integration (Bunge et al., 2005; Christoff et al., 2001; Ramnani and Owen, 2004; Wendelken and Bunge under review; Wendelken et al., 2007). Several lines of evidence led us to hypothesize that RLPFC would exhibit delayed maturation relative to VLPFC: (1) behavioral evidence that children have difficulty integrating multiple relations, as noted above, (2) structural MRI work suggesting that RLPFC exhibits adult-like gray matter density later in development than VLPFC (Gogtay et al., 2004; 0'Donnell et al., 2005), and (3) the hypothesis of a hierarchical organization in lateral PFC (Badre and D'Esposito, 2007; Bunge and Zelazo, 2006; Christoff and Gabrieli, 2002), with RLPFC further processing inputs from more posterior regions in PFC (Bunge et al., 2005).

For left VLPFC, no systematic differences were observed between children and adults. However, across the 6-13 age range, older children recruited left VLPFC and other regions more strongly than younger children during processing of single semantic relations. Both adults and children showed the same basic pattern of greater VLPFC activation on analogy vs. semantic trials, a pattern that was expected because participants had to engage in more extensive semantic processing on analogy trials than on semantic trials. These results provide evidence for a strengthened semantic network between ages 6 and 13 .

For children, increased RLPFC activation with age from 6 to 13 was observed bilaterally for semantic trials, and in left RLPFC for analogy trials. For adults, individuals who performed the analogy trials most accurately showed the greatest differential engagement in RLPFC for analogy $>$ semantic trials. Thus, although neither group consistently engaged RLPFC, older children were more likely to recruit this area than younger children, and better-performing adults were more likely to do so than worse-performing adults.

The fact that RLPFC was increasingly engaged from age $6-13$ on semantic trials was interesting, because this region is not strongly engaged by adults during the consideration of single relations (Christoff et al., 2001; Crone et al., in press). This finding suggests that RLPFC involvement in analogical reasoning goes through two developmental stages: first it is increasingly engaged during the processing of relations; then, by adulthood - particularly for better-performing adults - it becomes more specifically engaged during the processing and/or integration of multiple relations. In other words, these findings suggest that 
RLPFC is first incorporated into the relational processing network, as it is increasingly engaged from $6-13$, and that its role is refined during adolescence, particularly for adults who are most facile with these analogies. A similar account has been put forth with respect to changes in working memory and cognitive control circuitry over childhood and adolescence (Luna and Sweeney, 2004; Scherf et al., 2006).

A 4-second shift in the peak of RLPFC activation was observed for children relative to adults in both left and right RLPFC. This is a dramatic latency shift that we have not observed previously in our developmental fMRI research, either in RLPFC or elsewhere in the brain. Examination of the timecourse of motor cortical activation strongly suggested that, for children, the RLPFC response took place after the motor response. Thus, these regions were engaged by children, but not in time to process or integrate relations and influence the decision on the current trial. This finding is likely to explain, at least in part, why children performed disproportionately worse on analogy trials than adults.

In the adult cognitive neuroscientific literature, RLPFC has been strongly implicated in fluid reasoning. Studies of patients with early stages of frontotemporal dementia preferentially affecting the frontal lobes, have revealed deficits in their ability to integrate relations on a transitive inference task and RPM (Morrison et al., 2004; Waltz et al., 1999). RLPFC has also been consistently implicated in fMRI studies of reasoning, including the RPM task (Christoff et al., 2001; Kroger et al., 2002) and verbal propositional analogy tasks (Bunge et al., 2005; Green et al., 2006; Wendelken et al., 2007). In the RPM task, RLPFC has been found to be engaged more strongly on analogy problems - in which participants must jointly process two dimensions of change in the visual arrays - than on either 0-relational or 1-relational problems (Christoff et al., 2001; Crone et al., in press). In the verbal propositional analogy task (Bunge et al., 2005; Wendelken et al., 2007), RLPFC is engaged when participants must consider whether two semantic relations are analogous (e.g., "shoe is to sock as glove is to hand?").

Thus, these studies of reasoning in the visuospatial and verbal domains show that RLPFC is modulated by the number of mental relations to be considered, i.e., relational complexity. In addition to being engaged on fluid reasoning tasks, RLPFC is also engaged by numerous other cognitive tasks. However, a number of researchers have argued that a basic task requirement that drives RLPFC is the need to jointly consider or integrate several distinct mental relations (see Bunge et al., 2005; Christoff and Gabrieli, 2002; Ramnani and Owen, 2004). The joint consideration of distinct representations may well underlie the ability to abstract information from the environment. Indeed, it has been argued that all fluid intelligence problems involve abstraction (Garlick and Sejnowski, 2006). As such, RLPFC may carry out computations that are critical for fluid reasoning. Changes in the function of RLPFC over childhood and adolescence may contribute to improvements in this ability, and individual differences in RLPFC functioning may explain, at least in part, why some people have a greater capacity for fluid reasoning than others.

\section{CONCLUSION}

This initial fMRI study of analogical reasoning in children and adults showed that children engaged a similar network of brain regions to adults when processing one semantic relationship, but did not exhibit much additional brain activation during the integration of two semantic relationships on the more difficult analogy trials. A dramatic difference in the timecourse of activation between children and adults was observed in RLPFC, such that children - even the oldest ones, who engaged this region more strongly than younger children - engaged this region only after selecting a response, suggestive of impulsive responding. Children were more likely than adults to select semantically related distracters on the analogy problems, which contributed to their worse performance on these problems. These behavioral and brain imaging results are consistent with the idea that improved response inhibition would enhance fluid reasoning ability. In follow-up research, we are evaluating the effectiveness of fluid reasoning training in children.

\section{Semantic > Fixation}

Adults
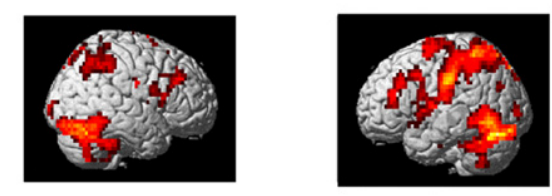

Children
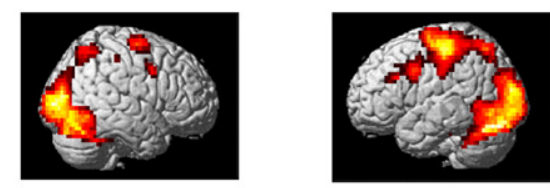

\section{Analogy $>$ Semantic}

Adults
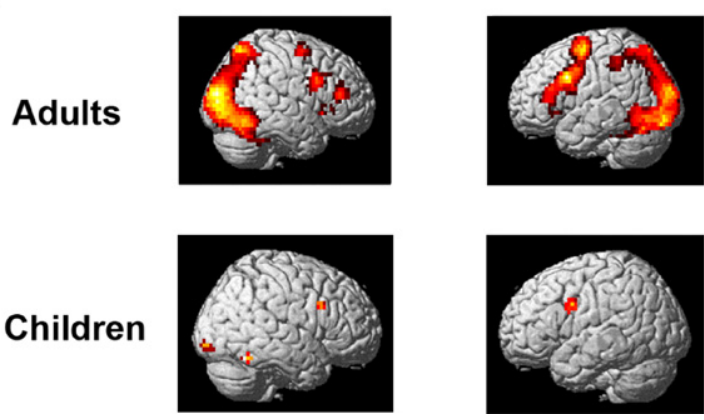

Figure S1. Whole-brain contrasts are displayed separately for adults and children.

\section{CONFLICT OF INTEREST STATEMENT}

The authors declare that the research was conducted in the absence of any commercial or financial relationships that could be construed as a potential conflict of interest.

\section{ACKNOWLEDGEMENTS}

The authors thank Pedro Paz-Alonso and Michael Souza for assistance with data analysis, and Chelsea Spitze for help creating the stimuli. Funding for this project was provided by R01NS057146 (S.A.B. \& E.F.) We thank Robert $G$. Morrison and two anonymous reviewers for helpful input on the initial submission.

\section{SUPPLEMENTARY MATERIAL}

See Figure S1.

\section{REFERENCES}

Badre, D., and D'Esposito, M. (2007). Functional magnetic resonance imaging evidence for a hierarchical organization of the prefrontal cortex. J. Cogn. Neurosci. 19, 2082-2099.

Blair, C. (2006). How similar are fluid cognition and general intelligence? A developmental neuroscience perspective on fluid cognition as an aspect of human cognitive ability. Behav. Brain Sci. 29, 109-125.

Braver, T. S., and Bongiolatti, S. R. (2002). The role of frontopolar cortex in subgoal processing during working memory. Neuroimage 15, 523-536.

Brown, A. (1990). Domain-specific principles affect learning and transfer in children. Cogn. Sci. 14, 107-133.

Bunge, S. A., Wendelken, C., Badre, D., and Wagner, A. D. (2005). Analogical reasoning and prefrontal cortex: evidence for separable retrieval and integration mechanisms. Cereb. Cortex 15, 239-249.

Bunge, S. A., and Zelazo, P. D. (2006). A brain-based account of the development of rule use in childhood. Curr. Dir. Psychol. Sci. 15, 118-121.

Burgund, E. D., Kang, H. C., Kelly, J. E., Buckner, R. L., Snyder, A. Z., Petersen, S. E., and Schlaggar, B. L. (2002). The feasibility of a common stereotactic space for children and adults in fMRI studies of development. Neuroimage 17, 184-200.

Carroll, J. (1997). Psychometrics, intelligence, and public perception. Intelligence 24, 25.

Cattell, R. B. (1971). Abilities: Their Structure, Growth and Action. Boston, MA, Houghton-Mifflin. 
Cattell, R. B. (1987). Intelligence: Its Structure, Growth and Action. Amsterdam, North-Holland Publishing Co.

Christoff, K., and Gabrieli, J. D. E. (2002). The frontopolar cortex and human cognition evidence for a rostrocaudal hierarchical organization within the human prefrontal cortex. Psychobiology 28, 168-186.

Christoff, K., Prabhakaran, V., Dorfman, J., Zhao, Z., Kroger, J. K., Holyoak, K. J., and Gabrieli, J. D. (2001). Rostrolateral prefrontal cortex involvement in relational integration during reasoning. Neuroimage 14, 1136-1149.

Christoff, K., Ream, J. M., Geddes, L. P., and Gabrieli, J. D. (2003). Evaluating self-generated information: anterior prefrontal contributions to human cognition. Behav. Neurosci. 117, 1161-1168.

Crone, E. A., Wendelken, C., van Leijenhorst, L., Honomichl, L. D., Christoff, K., and Bunge, S. A. (in press). Neurocognitive development of relational reasoning. Dev. Sci.

Ferrer, E., and McArdle, J. J. (2004). An experimental analysis of dynamic hypotheses about cognitive abilities and achievement from childhood to early adulthood. Dev. Psychol. 40, 935-952.

Ferrer, E., McArdle, J. J., Shawitz, B. A., Holahan, J. N., Marchione, K., and Shawitz, S. E. (2007). Longitudinal models of developmental dynamics between reading and cognition from childhood to adolescence. Dev. Psychol. 43,1460-1473.

Garlick, D., and Sejnowski, T. (2006). There is more to fluid intelligence than working memory capacity and executive function. Behav. Brain Sci. 29, 134-135.

Gentner, D. (1988). Metaphor as structure mapping: the relational shift. Child Dev. 59 , 47-59.

Gilhooly, K. J., and Logie, R. H. (1980). Age-of-acquisition, imagery, concreteness, familiarity, and ambiguity. Behav. Res. Methods Instrum. 12, 395-427.

Gogtay, N., Giedd, J. N., Lusk, L., Hayashi, K. M., Greenstein, D., Vaituzis, A. C., Nugent T. F. III, Herman, D. H., Clasen, L. S., Toga, A.W. et al. (2004). Dynamic mapping of human cortical development during childhood through early adulthood. Proc. Natl. Acad. Sci. U.S.A. 101, 8174-8179.

Goswami, U., and Brown, A. L. (1989). Melting chocolate and melting snowmen: analogical reasoning and causal relations. Cognition 35, 69-95.

Gray, J. R., Chabris, C. F., and Braver, T. S. (2003). Neural mechanisms of general fluid intelligence. Nat. Neurosci. 6, 316-322.

Green, A. E., Fugelsang, J. A., Kraemer, D. J., Shamosh, N. A., and Dunbar, K. N. (2006) Frontopolar cortex mediates abstract integration in analogy. Brain Res. 1096, 125-137.

Holyoak, K. J., Junn, E. N., and Billman, D. 0. (1984). Development of analogical problemsolving skill. Child Dev. 55, 2042-2055.

Horn, J. L. (1988). Thinking about human abilities. In Handbook of Multivariate Experimental Psychology, J. R. Nesselroade and R. B. Catell, eds. (New York, NY, Academic Press), pp. 645-685.

Horn, J., and Cattell, R. B. (1967). Age differences in fluid and crystallized intelligence. Acta Psychol. 26, 107-129.

Inhelder, B., and Piaget, J. (1958). The Growth of Logical Thinking from Childhood to Adolescence: An Essay on the Construction of Formal Operational Structures. New York, NY, Basic Books.

Kang, H. C., Burgund, E. D., Lugar, H. M., Petersen, S. E., and Schlaggar, B. L. (2003). Comparison of functional activation foci in children and adults using a common stereotactic space. Neuroimage 19, 16-28.

Kroger, J. K., Sabb, F. W., Fales, C. L., Bookheimer, S. Y., Cohen, M. S., and Holyoak, K. J. (2002). Recruitment of anterior dorsolateral prefrontal cortex in human reasoning: a parametric study of relational complexity. Cereb. Cortex 12, 477-485.
Luna, B., and Sweeney, J. A. (2004). The emergence of collaborative brain function: FMRI studies of the development of response inhibition. Ann. N. Y. Acad. Sci. 1021 296-309.

Lynn, R., Allik, J., and Irwing, P. (2004). Sex differences on three factors identified in Raven's Standard Progressive Matrices. Intelligence 32, 411-424

McArdle, J. J. (2001). A latent difference score approach to longitudinal dynamic structural analysis. In Structural Equation Modeling: Present and Future. A Festschrift in Honor of Karl Jöreskog, R. Cudeck, S. D. Toit and D. Sörbom, eds (Lincolnwood, IL, Scientific Software International), pp. 342-380.

McArdle, J. J., and Woodcock, J. R. (1998). Human Cognitive Abilities in Theory and Practice. Mahwah, NJ, Lawrence Erlbaum Associates.

Morrison, R. G., Krawczyk, D. C., Holyoak, K. J., Hummel, J. E., Chow, T. W., Miller, B. L., and Knowiton, B. J. (2004). A neurocomputational model of analogical reasoning and its breakdown in frontotemporal lobar degeneration. J. Cogn. Neurosci. 16, 260-271.

O'Donnell, S., Noseworthy, M. D., Levine, B., and Dennis, M. (2005). Cortical thickness of the frontopolar area in typically developing children and adolescents. Neuroimage 24, 948-954.

Piaget, J., Montangero, J., and Billeter, J. (1977). La formation des correlates. In Recherches sur l'abstraction reflechissante I, J. Piaget, ed (Paris, Presses Universitaires de France), pp. 115-129.

Pierce, K. A., and Gholson, B. (1994). Surface similarity and relational similarity in the development of analogical problem solving: Isomorphic and nonisomorphic transfer. Dev. Psychol. 30, 724-737.

Ramnani, N., and Owen, A. M. (2004). Anterior prefrontal cortex: insights into function from anatomy and neuroimaging. Nat. Rev. Neurosci. 5, 184-194.

Richland, L. E., Morrison, R. G., and Holyoak, K. J. (2006). Children's development of analogical reasoning: insights from scene analogy problems. J. Exp. Child Psychol. 94, 249-273.

Scherf, K. S., Sweeney, J. A., and Luna, B. (2006). Brain basis of developmental change in visuospatial working memory. J. Cogn. Neurosci. 18, 1045-1058.

Singer-Freeman, K. E., and Goswami, U. (2001). Does half a pizza equal half a box of chocolates? Proportional matching in an analogy task. Cogn. Dev. 16, 811-829.

Sternberg, R. J., and Downing, C. J. (1982). The development of higher-order reasoning in adolescence. Child Dev. 53, 209-221.

Sternberg, R. J., and Nigro, G. (1980). Developmental patterns in the solution of verbal analogies. Child Dev. 51, 27-38.

Thesen, S., Heid, O., Mueller, E., and Schad, L. R. (2000). Prospective acquisition correction for head motion with image-based tracking for real-time fMRI. Magn. Reson Med. 44, 457-465.

Tunteler, E., and Resing, W. C. (2002). Spontaneous analogical transfer in 4-year-olds: a microgenetic study. J. Exp. Child Psychol. 83, 149-166.

Wagner, A. D., Pare-Blagoev, E. J., Clark, J., and Poldrack, R. A. (2001). Recovering meaning: left prefrontal cortex guides controlled semantic retrieval. Neuron 31 , 329-338.

Waltz, J. A., Knowlton, B. J., Holyoak, K. J., Boone, K. B., Mishkin, F. S., de Menenzes Santos, M., Thomas, C. R., and Miller, B. L. (1999). A system for relational reasoning in human prefrontal cortex. Psychol. Sci. 10, 119-125.

Wendelken, C., and Bunge, S. A. (under review). Relational processing: distinct contributions of anterior prefrontal cortex and the hippocampus.

Wendelken, C., Nakhabenko, D., Donohue, S. E., Carter, C. S., and Bunge, S. A. (2007). "Brain Is to Thought as Stomach Is to ??": investigating the role of rostrolateral prefrontal cortex in relational reasoning. J. Cogn. Neurosci. 20, 1-12 